\title{
AVALIAÇÃO DOS EFEITOS DE PRÉ-TRATAMENTOS DA MADEIRA DE Eucalyptus benthamii Maiden \& Cambage NO GRAU DE COMPATIBILIDADE COM CIMENTO PORTLAND ${ }^{1}$
}

\begin{abstract}
Vinicius Castro², Roberto Daniel Araújo², Carlos Parchen ${ }^{3}$ e Setsuo Iwakiri ${ }^{4}$
RESUMO - O objetivo deste trabalho foi avaliar o efeito de cinco diferentes tipos de pré-tratamento na compatibilidade entre a madeira do Eucalyptus benthamii e o cimento Portland. A madeira foi pré-tratada com água fria, água quente, hidróxido de sódio, cloreto de cálcio ou hidróxido de cálcio. O grau de compatibilidade foi determinado através do estudo do calor de hidratação nas primeiras 24 h e pela resistência à compressão axial após 28 dias dos compósitos formados por cimento e pó de madeira na relação de peso de 13,3:1. A resistência à compressão axial após 28 dias também foi avaliada em compósitos formados com cimento e partículas de madeira na relação 2,75:1. Os resultados dos testes feitos com a madeira em pó indicaram que a adição de $3 \%$ de cloreto de cálcio é o tratamento mais eficiente para reduzir a capacidade de inibição da espécie, enquanto o tratamento do pó da madeira com hidróxido de cálcio afetou negativamente a compatibilidade. Entretanto, a combinação do aditivo cloreto de cálcio com partículas carbonatadas, pelo tratamento com hidróxido de cálcio, foi o que apresentou a maior resistência à compressão axial nos compósitos feitos na relação cimento:madeira de 2,75:1. A madeira de Eucalyptus benthamii apresentou potencial para a fabricação de painéis madeira-cimento. O uso da metodologia de carbonatação das partículas em solução de hidróxido de cálcio também é viável como pré-tratamento, independentemente dos resultados apontados pelos métodos tradicionais de medição do grau de compatibilidade.
\end{abstract}

Palavras-chave: Eucalyptus benthamii; Painéis madeira-cimento; Compatibilidade.

\section{EVALUATION OF PRETREATMENT EFFECTS OF Eucalyptus benthamii Maiden \& Cambage WOOD ON THE COMPATIBILITY DEGREE WITH PORTLAND CEMENT}

\begin{abstract}
This study aimed to evaluate the effect of five different types of pretreatment on the compatibility between Eucalyptus benthamii wood and Portland cement. The wood was pretreated with cold water, hot water, sodium hydroxide, calcium chloride or calcium hydroxide. Compatibility degree was determined through the study of the heat of hydration on the first 24 hours, and the axial compression strength, after 28 days of the composites made with a 13.3:1 weight ratio for cement and wood powder. The axial compression strength after 28 days was also evaluated for composites made with a 2.75:1 weight ratio for cement and wood particles. The results of the tests made with wood powder indicated that the addition of $3 \%$ of calcium chloride is the most efficient treatment to reduce the specie inhibition, while the treatment of the wood powder with calcium hydroxide had a negative effect in the compatibility. However, the combination of calcium chloride additive with carbonated particles through calcium hydroxide treatment presented the highest axial compression strength within the composites made with a cement:wood weight ratio of 2.75:1. The wood of Eucalyptus benthamii has potential for wood-cement panels manufacture. The use of the methodology of particles carbonation
\end{abstract}

\footnotetext{
${ }^{1}$ Recebido em 07.05.2013 aceito para publicação em 28.07.2014.

${ }^{2}$ Programa de Pós-Graduação em Engenharia Florestal na Universidade Federal do Paraná, UFPR, Brasil. E-mail: <vinnie.castro@gmail.com>e <rdanielrda@gmail.com>.

${ }^{3}$ Departamento de Construção Civil, Universidade Federal do Paraná, Brasil. E-mail: <parchen@ufpr.br>.

${ }^{4}$ Departamento de Engenharia e Tecnologia Florestal, Universidade Federal do Paraná, Brasil. E-mail: <setsuo@ufpr.br> .
} 
in a solution of calcium hydroxide is also viable as pretreatment, independently of the results presented by traditional methods of measuring the compatibility degree.

Keywords: Eucalyptus benthamii; Wood-cement panels; Compatibility.

\section{INTRODUÇÃO}

O Eucalyptus benthamii Maiden et Cambage é uma árvore moderadamente alta, atingindo $36 \mathrm{~m}$ de altura e diâmetro de $50 \mathrm{~cm}$, originária da Austrália. Foi introduzido no Brasil pela Embrapa/Florestas em 1988, no Município de Colombo, PR. Esse povoamento foi formado por famílias misturadas de 10 matrizes da procedência geográfica Wentworth Falls, Austrália, e mostrou-se resistente à geada, de rápido crescimento, boa forma de fuste e alta homogeneidade, tornandoo excelente opção para reflorestamento em regiões onde ocorrem geadas frequentes e severas, como no Sul do Brasil (GRAÇA et al., 1999). Para ampliar a base genética da população no Brasil, em 2005 a Embrapa Florestas importou 36 lotes de sementes, na forma de progênies de polinização aberta e, ainda, dois lotes adicionais, formados a partir da mistura de sementes colhidas em árvores pertencentes a duas populações naturais (PALUDZYSZYN FILHO et al., 2006).

A madeira de Eucalyptus benthamii é moderadamente dura (densidade básica de $0,47 \mathrm{~g} / \mathrm{cm}^{3}$ ), cerne e alburno distintos, sendo o cerne de cor marromavermelhada e o alburno rosado. Apresenta textura fina a média, grã-irregular, cheiro e gosto imperceptíveis e superfície levemente brilhante (NISGOSKI et al., 1998).

O uso da madeira de Eucalyptus benthamii vem sendo estudada para diversas finalidades, como uso da madeira termicamente tratada para pallets (PIRRAGLIA et al., 2012), produção de celulose (ALVES et al., 2011), energia (LIMA et al., 2007), compensados (MARTINS et al., 2011) e EGP (MARTINS, 2011). Uma possível nova destinação para uso dessa madeira é a produção de painéis madeira-cimento, uma vez que exigem madeiras particuladas que podem ser obtidas de árvores de pequenos diâmetros ou resíduos do processamento da madeira sólida.

O desenvolvimento das indústrias de painéis madeira-cimento teve início nos anos de 1930, embora a maior parte das inovações tenha ocorrido nos últimos 40 anos (FRYBORT et al., 2008). Esse tipo de compósito combina vantagens tanto dos componentes orgânicos quanto inorgânicos. A incorporação do cimento leva a um painel com alta estabilidade dimensional, alta resistência aos agentes biodegradadores e resistência ao fogo, ao mesmo tempo que a mistura com madeira resulta em painéis mais leves em relação ao concreto, de maior trabalhabilidade e com boas características acústicas e de isolamento térmico (MOSLEMI, 1989).

Entre as principais dificuldades na produção deste tipo de painel está, porém, a seletividade das espécies de madeiras utilizadas na mistura (MOSLEMI; PFISTER, 1987). Segundo Latorraca et al. (1999), os componentes orgânicos afetam tanto a possível interação entre a madeira e o cimento quanto as interações internas do próprio cimento, resultando em painéis de qualidade inferior. A incompatibilidade da mistura madeira-cimento é normalmente associada aos extrativos (JORGE et al., 2004).

Diferentes métodos são empregados para minimizar o efeito da incompatibilidade entre cimento e madeira, como o uso de preservativos de madeira, armazenamento prolongado da madeira, extração dos açúcares solúveis em água quente e fria, mistura com aditivos químicos ou aceleradores em solução diluída, como hidróxido de sódio $(\mathrm{NaOH})$ e cloreto de cálcio $\left(\mathrm{CaCl}_{2}\right)(\mathrm{MOSLEMI}$ et al., 1983).

O teor de extrativos do Eucalyptus benthamii pode variar de acordo com a idade da árvore. Para amostras retiradas de um plantio de quatro anos, o teor de extrativos é de 6\%, enquanto em um plantio de sete anos o teor foi reduzido para, aproximadamente, a metade (3,2 \pm 0,3\%) (PEREIRA et al., 2000). Em outro estudo, a solubilidade dos extrativos em acetona foi tida como $2,25 \%$, porém com o detalhe de que os extrativos hidrofóbicos solúveis em diclorometano foi de apenas 0,2\% (ALVES et al., 2011).

Semple et al. (2002a) avaliaram a compatibilidade de oito espécies de Eucalyptus de clima temperado, entre elas o Eucalyptus benthamii, e do cimento Portland. Essa espécie apresentou um dos valores mais baixos de compatibilidade, com redução da taxa de hidratação do cimento de aproximadamente 2,2 para $1,01^{\circ} \mathrm{C} / \mathrm{h}$. 
Este trabalho teve como objetivo avaliar os efeitos de cinco métodos de pré-tratamento de partículas de madeira de Eucalyptus benthamii no grau de compatibilidade em mistura com cimento Portland.

\section{MATERIAL E MÉTODO}

A espécie utilizada nesta pesquisa foi Eucalyptus benthamii Maiden et Cambage de 13 anos de idade, procedente de plantio experimental localizado no Município de Palmeira, Estado de Santa Catarina. O material foi obtido na forma de tábuas, as quais foram seccionadas em blocos para geração de partículas no picador de disco. Após a moagem em moinho de martelo, as partículas foram classificadas em peneiras, sendo selecionadas aquelas que passaram na peneira com $0,84 \mathrm{~mm}$ de abertura e ficaram retidas na peneira de $0,5 \mathrm{~mm}$. O aglutinante empregado foi o cimento Portland tipo CP II. Os pré-tratamentos aos quais as partículas foram submetidas estão expressos na Tabela 1.

Após os pré-tratamentos, as partículas foram lavadas em água corrente e secas em estufa até o teor de umidade médio de $3 \%$, com exceção do tratamento com cal (T6). No tratamento T6, as partículas foram pré-tratadas em solução de hidróxido de cálcio (com 21,3\% de óxido de cálcio $(\mathrm{CaO}))$ em uma proporção de 1:1 em relação ao peso. As partículas não foram lavadas em água corrente, tendo sido submetidas à secagem ainda impregnadas com a solução. O hidróxido de cálcio tem a propriedade de endurecer lentamente ao ar, unindo fortemente as partículas sólidas. Trata-se de reação química de carbonatação, ou seja, o hidróxido de cálcio $\left(\mathrm{Ca}(\mathrm{OH})_{2}\right)$ em contato com o dióxido de carbono da atmosfera forma

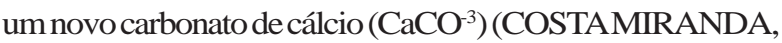
2009), que é o produto branco que encobre as partículas.

\subsection{Teste de hidratação}

Água destilada (90,5 mL) foi misturada com 200 g de cimento e $15 \mathrm{~g}$ de madeira. A quantidade de água foi baseada no experimento de Weatherwax e Tarkow

Tabela 1 - Plano experimental.

Table 1 - Experimental plan.

\begin{tabular}{cl}
\hline Tratamento & Pré-tratamento das partículas \\
\hline T 1 & Sem tratamento \\
T 2 & 24 h de imersão em água fria \\
T 3 & 6 h de imersão em água quente $\left(60{ }^{\circ} \mathrm{C}\right)$ \\
T 4 & 2 h de imersão em solução alcalina $(1 \% \mathrm{NaOH})$ \\
T 5 & Adição de $3 \%$ de $\mathrm{CaCl}_{2}$ \\
T 6 & Imersão em cal hidratada (relação $1: 1)$ \\
\hline
\end{tabular}

(1964), que sugere o uso de 2,7 mL de água por grama de madeira e um adicional de $0,25 \mathrm{~mL}$ de água por grama de cimento.

A mistura ocorreu em sacos de polietileno, e o tempo não excedeu 5 min; em seguida, foi colocada em um recipiente isolado térmica e hermeticamente fechado. Para a leitura das temperaturas, termopares tipo $\mathrm{J}$ foram conectados a um módulo condicionador de sinais analógicos, acoplado a uma placa de conversão analógico-digital. Foram realizadas leituras de temperatura a cada minuto, durante 24 h. Com base nos parâmetros obtidos, foram utilizados três diferentes métodos para determinar a inibição da cura do cimento induzida pela madeira:

$\mathbf{T}_{\mathrm{R}}$ - que é uma variação do método de Weatherwax e Tarkow (1964), sugerida por Olorunnisola (2008), em que a inibição indicada pela razão do tempo da cura da mistura, ou seja, o tempo para a reação atingir sua temperatura máxima; e pelo tempo da cura do cimento, sem a adição de madeira. As madeiras podem ser classificadas em três categorias, de acordo com o seu valor de $\mathbf{T}_{\mathbf{R}}$ : compatíveis $(\leq 1,5)$; aceitáveis (valores entre 1,5 e 2), ou seja, necessitam de um pré-tratamento; e incompatíveis ( $\geq 2$ ) (OLORUNNISOLA, 2008).

Fator- $\mathrm{C}_{\mathrm{A}}$ - Método sugerido por Hachmi e Moslemi (1989), cujo fator é a razão entre a área do gráfico da reação com mistura de madeira e a área do gráfico da reação do cimento com água, sem adição de madeira. Para determinação desse fator, é considerado o tempo entre 3,5 h após a mistura e 24 h. Valores de fator$\mathrm{C}_{\mathrm{A}}$ acima de 68 indicam uma madeira compatível com o cimento, sem necessidade de pré-tratamento, enquanto valores abaixo de 28 representam espécies incompatíveis. Valores entre 28 e 68 indicam a necessidade de um prétratamento da madeira (HACHMI; MOSLEMI, 1989).

Índice de Inibição (II) - Este índice foi inicialmente sugerido por Hofstrand et al. (1984) e leva em consideração a temperatura máxima da reação, o tempo para atingir essa temperatura e a taxa máxima de incremento de temperatura da reação. Com base nesses parâmetros, o índice é calculado pela fórmula:

$$
\begin{aligned}
& \mathrm{II}=100\left[\left(\mathrm{t}-\mathrm{t}^{\prime} / \mathrm{t}^{\prime}\right)\left(\mathrm{T}_{-} \text {' - T / T’) ( S'-S / S') }\right]\right. \\
& \text { em que: } \\
& \text { I = índice de inibição da cura do cimento (\%); } \\
& \text { t = tempo necessário para atingir a temperatura }
\end{aligned}
$$
máxima de hidratação do cimento da mistura de cimento, água e madeira (h);

Revista Árvore, Viçosa-MG, v.38, n.5, p.935-942, 2014

\section{Silf}


t' = tempo da temperatura máxima de hidratação do cimento da mistura cimento e água (h);

T' = temperatura máxima atingida para mistura cimento-água $\left({ }^{\circ} \mathrm{C}\right)$;

$\mathrm{T}=$ temperatura máxima atingida para cimentoágua e madeira $\left({ }^{\circ} \mathrm{C}\right)$;

$\mathrm{S}^{\prime}=\Delta \mathrm{T}^{\prime} / \Delta \mathrm{t}^{\prime}$ - máximo incremento no período de 1 h para mistura cimento-água; e

$\mathrm{S}=\Delta \mathrm{T} / \Delta \mathrm{t}-$ máximo incremento no período de $1 \mathrm{~h}$ para mistura cimento-água madeira.

A inibição da madeira à cura do cimento pode ser classificada em quatro categorias, de acordo com o índice de inibição: baixa (II < 10); moderada (II = 10 - 50); alta (II = 50 - 100); e extrema (II > 100) (OKINO et al., 2005).

\subsection{Teste de compatibilidade por compressão axial}

A relação cimento:madeira foi mantida em 13,3:1, como sugerido por Lee e Hong (1986). A quantidade de água utilizada na mistura seguiu a fórmula sugerida por Souza (1994 citado por LATORRACA, 2000), que é:

Agua $=0,43 \times$ cimento $+($ madeira $\times(0,3-$ teor de umidade da madeira))

A mistura foi, então, colocada em formas cilíndricas de PVC de 3,5 cm de diâmetro por $7 \mathrm{~cm}$ de comprimento. Os corpos de prova foram mantidos no cilindro por sete dias; após esse período, foram retirados da fôrma e deixados ao ar livre por 28 e 56 dias.

Os corpos de prova foram submetidos ao teste de compressão axial descrito na Norma NBR 7512 (ABNT, 1996).

\subsection{Teste de compressão axial de compósitos madeira- cimento}

Foram produzidos quatro corpos de prova cilíndricos com 3,5 cm de diâmetro e $7 \mathrm{~cm}$ de comprimento, para quatro tipos de compósitos: C1, partículas de madeira sem pré-tratamento; C2, partículas submetidas à extração em água quente $\left(60^{\circ} \mathrm{C}\right.$ por 6 h); C3, extração em solução alcalina (solução de 1\% de hidróxido de sódio por 2 h); e C4, partículas tratadas com cal hidratada (relação 1:1 em relação ao peso da madeira seca). Após o pré-tratamento, as partículas foram lavadas com água corrente e secas ao ar livre, com exceção do tratamento C4, em que as partículas foram secas sem serem lavadas, para promover a carbonatação pelo hidróxido de cálcio.

O método de produção de corpos de prova utilizado foi o de adensamento por vibro compactação sem prensa, sugerido por Parchen (2012). A mistura de cimento:madeira seguiu a relação 2,75: 1 e a quantidade de água sugerida por Souza (1994 citado por LATORRACA, 2000), sendo adicionados 3\% de cloreto de cálcio. A mistura foi, então, submetida à mesa vibratória com uma carga de impacto vertical equivalente a 22 da $\mathrm{N}$ a 3.000 RPM.

Os corpos de prova foram submetidos ao teste de compressão axial descrito na Norma NBR 7512 (ABNT, 1996), após 28 dias.

\section{RESULTADOS}

\subsection{Teste de hidratação}

O resultado dos testes de hidratação pode ser observado pelo gráfico apresentado na Figura 1, que indica o comportamento exotérmico da reação de cura do cimento nas $24 \mathrm{~h}$ iniciais.

A Tabela 2 mostra os parâmetros de hidratação do cimento puro em comparação com o cimento misturado com a madeira de Eucalyptus benthamii, assim como o grau de inibição indicado por três diferentes métodos.

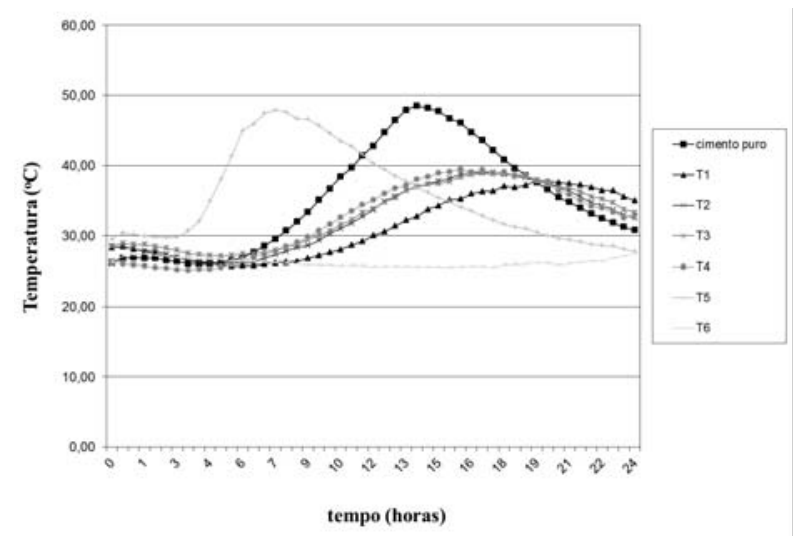

Figura 1 -Efeito dos pré-tratamentos da madeira de Eucalyptus benthamii sobre a hidratação inicial do cimento Portland tipo II.

Figure 1 - Effect of pretreated Eucalyptus benthamii wood on the initial hydration of the Portland cement type II. 
Tabela 2 - Efeito dos pré-tratamentos da madeira de Eucalyptus benthamii nas características de hidratação da mistura madeira-cimento .

Table 2 - Effect of pretreated Eucalyptus benthamii wood on the hydration characteristics of mixture wood-cement.

\begin{tabular}{ccccccc}
\hline & $\mathrm{T}_{\text {máx. }\left({ }^{\circ} \mathrm{C}\right)}$ & $\mathrm{t}$ máx. $(\mathrm{h})$ & $\mathrm{S}$ máx. $\Delta \mathrm{T} / \Delta \mathrm{t}\left({ }^{\circ} \mathrm{C} / \mathrm{h}\right)$ & $\mathrm{T}_{\mathrm{R}}$ & Fator-C $_{\mathrm{A}}(\%)$ & $\mathrm{II}(\%)$ \\
\hline Cimento & $48,96^{\mathrm{A}}$ & $14,42^{\mathrm{E}}$ & $4,35^{\mathrm{B}}$ & - & - & - \\
T 1 & $38,01^{\mathrm{B}}$ & $20,24^{\mathrm{B}}$ & $2,00^{\mathrm{CD}}$ & $1,40^{\mathrm{B}}$ & $86,55^{\mathrm{B}}$ & $4,87^{\mathrm{B}}$ \\
T 2 & $39,37^{\mathrm{B}}$ & $17,25^{\mathrm{CD}}$ & $2,38^{\mathrm{C}}$ & $1,20^{\mathrm{CD}}$ & $92,09^{\mathrm{B}}$ & $1,75^{\mathrm{BC}}$ \\
T 3 & $39,41^{\mathrm{B}}$ & $18,15^{\mathrm{C}}$ & $2,00^{\mathrm{CD}}$ & $1,26^{\mathrm{C}}$ & $91,51^{\mathrm{B}}$ & $2,87^{\mathrm{BC}}$ \\
T 4 & $39,91^{\mathrm{B}}$ & $16,76^{\mathrm{D}}$ & $2,20^{\mathrm{C}}$ & $1,16^{\mathrm{D}}$ & $91,40^{\mathrm{B}}$ & $1,52^{\mathrm{C}}$ \\
T 5 & $48,12^{\mathrm{A}}$ & $7,45^{\mathrm{F}}$ & $7,59^{\mathrm{A}}$ & $0,54^{\mathrm{E}}$ & $100,03^{\mathrm{A}}$ & $0,55^{\mathrm{C}}$ \\
T 6 & $27,49^{\mathrm{C}}$ & $23,82^{\mathrm{A}}$ & $1,03^{\mathrm{D}}$ & $1,65^{\mathrm{A}}$ & $70,84^{\mathrm{C}}$ & $21,79^{\mathrm{A}}$ \\
\hline
\end{tabular}

* Valores médios de três repetições.

** Médias seguidas pela mesma letra dentro da mesma coluna não se diferenciam estatisticamente, a 5\% de probabilidade, pelo teste de Tukey.

* Average values of three repetitions.

** Averages followed by the same letter do not differ from each other, according to the Tukey test at 5\%.

\subsection{Teste de compatibilidade por compressão axial}

Na Tabela 3 estão apresentados os valores da densidade e resistência à compressão axial dos corpos de prova cilíndricos produzidos para os seis tratamentos em estudo.

\subsection{Teste de compressão axial para compósitos madeira-cimento}

A Tabela 4 apresenta os valores da densidade e da resistência à compressão axial dos corpos de prova de madeira-cimento produzidos pelo método de vibração mecânica na relação cimento:madeira de 2,75:1. Uma vez que todos os testes de compatibilidade indicaram que o uso do aditivo cloreto de cálcio é o mais eficiente para diminuição da inibição da cura

Tabela 3 - Valores da densidade e resistência à compressão axial.

Table 3 - Values of density and axial compression strength.

\begin{tabular}{ccc}
\hline Tratamento & Densidade $\left(\mathrm{g} / \mathrm{cm}^{3}\right)$ & $\begin{array}{c}\text { Compressão axial } \\
(\mathrm{MPa})\end{array}$ \\
\hline T1 (56 dias) & $1,62^{\mathrm{A}}$ & $15,68(0,14)^{\mathrm{AB}}$ \\
T1 (28 dias) & $1,56^{\mathrm{BC}}$ & $16,72(0,08)^{\mathrm{A}}$ \\
T 2 & $1,54^{\mathrm{BC}}$ & $16,99(0,09)^{\mathrm{A}}$ \\
T 3 & $1,54^{\mathrm{C}}$ & $15,24(0,10)^{\mathrm{AB}}$ \\
T 4 & $1,53^{\mathrm{C}}$ & $11,79(0,13)^{\mathrm{C}}$ \\
T 5 & $1,58^{\mathrm{AB}}$ & $17,03(0,13)^{\mathrm{A}}$ \\
T 6 & $1,55^{\mathrm{BC}}$ & $12,93(0,24)^{\mathrm{BC}}$ \\
\hline
\end{tabular}

* Médias seguidas pela mesma letra dentro da mesma coluna não se diferenciam estatisticamente, a $5 \%$ de probabilidade, pelo teste de Tukey.

** Valores entre parênteses referem-se ao coeficiente de variação.

* Averages followed by the same letter do not differ from each other, according to the Tukey test at 5\%.

** Values between parentheses indicate the coefficient variation.
Tabela 4 - Valores da densidade e resistência à compressão axial de compósitos madeira-cimento.

Table 4 - Values of density and axial compression strength of the wood-cement composites.

\begin{tabular}{ccc}
\hline Tratamento & $\begin{array}{c}\text { Densidade } \\
\left(\mathrm{g} / \mathrm{cm}^{3}\right)\end{array}$ & $\begin{array}{c}\text { Compressão axial } \\
(\mathrm{MPa})\end{array}$ \\
\hline C1 & $0,88^{\mathrm{A}}$ & $4,20^{\mathrm{B}}(0,09)$ \\
$\mathrm{C} 2$ & $0,75^{\mathrm{B}}$ & $3,59^{\mathrm{B}}(0,17)$ \\
C3 & $0,83^{\mathrm{A}}$ & $3,51^{\mathrm{B}}(0,11)$ \\
C4 & $1,22^{\mathrm{C}}$ & $9,57^{\mathrm{A}}(0,23)$ \\
\hline
\end{tabular}

* Valores médios de quatro repetições.

** Médias seguidas pela mesma letra dentro da mesma coluna não se diferenciam estatisticamente, a 5\% de probabilidade, pelo teste de Tukey.

*** Valores entre parênteses referentes ao coeficiente de variação. * Average values of four repetitions.

** Averages followed by the same letter do not differ from each other, according to the Tukey test at 5\%.

*** Values between parentheses indicate the coefficient variation.

do cimento causada pela presença da madeira de Eucalyptus benthamii, e 3\% do aditivo foi misturado a todos os compósitos.

\section{DISCUSSÃO}

\subsection{Teste de hidratação}

Pode-se observar comportamento semelhante nas curvas da mistura da madeira de Eucalyptus benthamii sem tratamento (T1) e dos tratamentos por extração (T2, T3 e T4). O tratamento com aditivo cloreto de cálcio (T5) foi o único que antecipou a formação do cristal resistente silicato de cálcio hidratado (C-S-H) representado pelo pico de temperatura. Já no tratamento com cal (T6) não houve nenhuma alteração dentro do período de $24 \mathrm{~h}$.

Revista Árvore, Viçosa-MG, v.38, n.5, p.935-942, 2014 
Em todos os três índices avaliados, evidenciou-se que a inibição causada pela madeira pode ser eliminada com o uso de 3\% do aditivo cloreto de cálcio (T5). Entretanto, nos três métodos de avaliação o uso da solução de hidróxido de cálcio (T6) interfere negativamente no processo de cura inicial do cimento. Isso porque, nesse pré-tratamento, a cura do cimento não é somente inibida pela presença da madeira, mas também pela relação cal-cimento (GLASSER, 1997).

Nos pré-tratamentos para remoção de extrativos (T2, T3 e T4), os métodos não apresentaram definição clara na melhora significativa nos índices, embora haja tendência geral que permite indicar que o método de extração em água quente (T3) é o menos eficiente. O método $\mathrm{T}_{\mathrm{R}}$ e o II indicam a extração em solução alcalina (T4) como o melhor pré-tratamento de extração. Já pelo método do Fator- $\mathrm{C}_{\mathrm{A}}$ não há diferença entre esses três tratamentos e a testemunha (T1).

O índice de inibição (II) para o tratamento T1 indica que o Eucalyptus benthamii apresenta comportamento semelhante ao de outras espécies de eucaliptos. Latorraca (2000) determinou o índice de inibição de quatro espécies de Eucalyptus (E. robusta, E. urophylla, E. pellita e E. citriodora) e constatou que, em todas as espécies estudadas, os valores de II foram abaixo de $10 \%$, e apenas o E. robusta apresentou valor inferior a $5 \%$.

$\mathrm{O}$ fator- $\mathrm{C}_{\mathrm{A}}$ do Eucalyptus benthamii também foi superior ao encontrado na literatura para outras espécies de eucalipto (E. angus, E. horistes, E. loxophlebo, E. plenissima e E. polybractea). Entre essas espécies, o maior valor de compatibilidade foi o de E. horistes, com fator- $C_{A}$ de apenas 70,8\% (SEMPLE et al., 2002b).

\subsection{Teste de compatibilidade por compressão axial}

Os tratamentos apresentaram densidades semelhantes, com exceção do tratamento com 3\% de aditivo (T5), que apresentou valor estatisticamente superior aos tratamentos de extração com água quente (T3) e com hidróxido de sódio (T4). Também, podese observar a diferença estatística para densidade entre os corpos de prova com partículas de Eucalyptus benthamii sem tratamento prévio (T1) com diferentes tempos de cura. Após 56 dias, os corpos de prova apresentaram densidade superior a dos corpos de prova com apenas 28 dias de cura. Contudo, não houve diferença estatística nos valores de resistência à compressão dos corpos de prova após 28 ou 56 dias, comprovando que a adição de madeira não afetou o prazo de cura do cimento de 28 dias.

Comparado com o resultado de compressão axial de outras espécies de Eucalyptus apresentado por Latorraca (2000), o Eucalyptus benthamii apresentou resultado semelhante com o do Eucalyptus urophylla (16,38 MPa).

O tratamento pelo método de extração com hidróxido de sódio (T4) apresentou resultados favoráveis na maioria dos testes de inibição da cura inicial do cimento, porém no teste de compressão axial, após 28 dias de cura, ele se mostrou menos resistente do que os demais tratamentos.

\subsection{Teste de compressão axial para compósitos madeira-cimento}

A maior densidade dos compósitos C4 pode ser atribuída à maior quantidade de material inorgânico. Após a secagem da cal, há um ganho de cerca de 17\% do peso da partícula, graças à incorporação do hidróxido de cálcio.

Não houve diferença significativa nos valores de resistência à compressão axial entre os compósitos produzidos com partículas após a extração em água quente (C2), extração em solução de hidróxido de sódio (C3) e partículas sem pré-tratamento (C1). Esse comportamento está de acordo com os resultados de outras espécies de Eucalyptus. Beraldo e Carvalho (2004) também constataram que não há necessidade de aplicação de pré-tratamento com água quente para partículas de Eucalypitus grandis, citando o mesmo comportamento em trabalhos prévios com Eucalyptus citriodora.

Não se pode correlacionar o efeito dos prétratamentos na curva de hidratação inicial do cimento com a resistência da compressão axial, após 28 dias, para os compósitos produzidos com partículas carbonatadas pelo pré-tratamento com cal hidratada (C4). Os compósitos C4 foram os que apresentaram a maior resistência, e os métodos de determinação de compatibilidade apontaram que o tratamento com cal (T6) afetou negativamente a cura do cimento e, teoricamente, não seria recomendado para fabricação de painéis madeira-cimento. A explicação para esse fenômeno pode estar no fato de que a carbonatação é uma reação que ocorre na superfície das partículas. 
Os métodos tradicionais de determinação de compatibilidade usam a madeira em forma de pó, com maior área superficial do que as partículas usadas na formação do compósito.

Efeito semelhante foi relatado por Zucco (1999), que estudou o efeito da granulometria da casca de arroz tratada com hidróxido de cálcio na cura inicial do cimento. Esse autor relatou que a inibição do material tratado passante na peneira de $0,15 \mathrm{~mm}$ era maior do que o em estado natural. Porém, para partículas maiores, passantes na peneira de 0,3 mm, esse comportamento se inverteu, e o tratamento passou a afetar positivamente a compatibilidade.

\section{CONCLUSÃO}

A madeira de Eucalyptus benthamii apresenta potencial para ser utilizada na fabricação de painéis de madeira-cimento. Entre os pré-tratamentos, o que se mostrou mais eficiente para reduzir a inibição, que a madeira dessa espécie causa na cura inicial do cimento, foi a adição de 3\% do cloreto de cálcio.

Considerando os métodos tradicionais de determinação do grau de compatibilidade entre madeira e cimento, não há necessidade de nenhum prétratamento, uma vez que todos indicaram valores que classificam a madeira de Eucalyptus benthamii como naturalmente compatível com o cimento.

A combinação do aditivo cloreto de cálcio com partículas carbonatadas, pelo pré-tratamento com solução em hidróxido de cálcio, foi a que apresentou a maior resistência à compressão axial, após 28 dias, nos compósitos feitos na relação cimento:madeira de 2,75:1. Esse resultado indica que a metodologia de cabonatação com cal hidratada é viável como pré-tratamento, mesmo que a cura inicial do cimento seja afetada negativamente, como foi indicado pelos métodos de compatibilidade conduzidos com pó de madeira, dentro da relação cimento:madeira de 13,3:1.

\section{REFERÊNCIAS}

ALVES, I. C. N. et al. Caracterização tecnológica da madeira de Eucalyptus benthamii para produção de celulose kraft. Ciência Florestal, v.21, n.1, p.167-174, 2011

ASSOCIAÇÃO BRASILEIRA DE NORMAS TÉCNICAS - ABNT. NBR 7215: Cimento Portland - determinação da resistência à compressão. Rio de Janeiro: 1996.
BERALDO, A. L.; CARVALHO, J. V. Compósito Eucalyptus grandis - Cimento Portland. Scientia Forestalis, v.65, p.150-161, 2004.

COSTA MIRANDA, L. M. C. Estudo comparativo entre argamassa de revestimento à base de cimento com adição de cal hidráulica e da cal hidratada. 2009. 78f. Dissertação (Mestrado em Engenharia Civil) - Universidade de Trás-osMontes e Alto Douro, Vila Real, 2009.

FRYBORT, S. et al. Cement bonded composites - a mechanical review. Bioresources, v.3, n.2, p.602-623, 2008.

GLASSER, F. P. Fundamental aspects of cement solidification and stabilisation. Journal of Hazardous Material, n.52, p.151-170, 1997.

GRAÇA, M. E. C.; SHIMIZU, J. Y.; TAVARES, F. $\mathrm{R}$. Capacidade de rebrota e de enraizamento de Eucalyptus benthamii. Boletim de Pesquisa Florestal, n.39, p.135-138, 1999.

HACHMI, M.; MOSLEMI, A. A. Correlation between wood-cement compatibility and wood extractives. Forest Products Journal, v.39, n.6, p.55-58, 1989.

HOFSTRAND, A. D.; MOSLEMI, A. A.; GARCIA J. F. Curing characteristics of wood particle from nine northern Rocky Mountain species mixed with Portland Cement. Forest Products Journal, v.34, n.2, p.57-61, 1984.

JORGE, F. C.; PEREIRA, C.; FERREIRA, J. M. F. Wood-cement composites: a review. Holz als Roh und Werkstoff, v.62. p.370-377, 2004.

LATORRACA J. V.; IWAKIRI S.; LELIS R. C. Efeito Inibidor de cinco espécies Florestais sobre a cura do compósito cimento-madeira. Floresta e Ambiente, v.6, n.1, p.76-82, 1999.

LATORRACA, J. V. F. Eucalyptus spp. na produção de painéis de cimentomadeira. 2000. 191f. Tese (Doutorado em Ciências Florestais) - Universidade Federal do Paraná, Curitiba, 2000.

LEE A. W. C.; HONG, Z. Compressive strength of cylindrical samples as an indicator of woodcement compatibility. Forest Product Journal, v.36. n.11/12, p.87-90, 1986

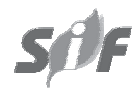

Revista Árvore, Viçosa-MG, v.38, n.5, p.935-942, 2014 
LIMA, E. A. et al. Caracterização individual de árvores de Eucalyptus benthamii para uso energético. Colombo: Embrapa Florestais, 2007. 25p. (Boletim de Pesquisa e Desenvolvimento, 35)

MARTins, S. A. Adequação tecnológica da madeira de Eucalyptus benthamii Maiden et Cambage Para a produção de painéis colados lateralmente (PCL). 2011. 96f. Dissertação (Mestrado em Ciências Florestais) - Universidade de Brasília, Brasília, 2011.

MARTINS, S. A.; CUNHA, A. B.; OLIVEIRA, J. Avaliação da qualidade da colagem de painéis compensados de Eucalyptus benthamii Maiden et Cambage. In: CONGRESSO IBERO-LATINO AMERICANO DA MADEIRA NA CONSTRUÇÃO, 1., 2011, Coimbra. Proceedings... Coimbra: Departamento de Engenharia Civil da FCTUC, 2011.

MOSLEMI, A. A.; GARCIA, J. F.; HOFSTRAND, A. D. Effect of various treatments and additives on wood-Portland cement-water systems. Wood and Fiber Science, v.15, n.2. p.164-176, 1983.

MOSLEMI, A. A.; PFISTER, S. C. The influence of cement/wood ratio and type on bending strength and dimensional stability of wood-cement composite panels. Wood and Fiber Science, v.19, n.2, p.165-175, 1987.

MOSLEMI, A. A. Wood-cement panel products: coming of age. In: MOSLEMI, A. A.; HAMEL, M. P. Fiber and particleboards bonded with inorganic binders. Madison: Forest Product Research Society, 1989. v.1. p.12-18.

NISGOSKI, S.; MUNIZ, G. I. B.; KLOCK, U. Caracterização anatômica da madeira de Eucalyptus benthamii Maiden et Cambage. Ciência Florestal, v.8, n.1, p.67-76, 1998.

OKINO, E. Y. A. et al. Physico-mechanical properties and decay resistance of Cupressus spp. cement-bonded particleboards. Cement and Concrete Composites, v.27, p.333-338, 2005.

Revista Árvore, Viçosa-MG, v.38, n.5, p.935-942, 2014
OLORUNNISOLA, A. O. Effects of pre-treatment of rattan (Laccosperma secundiflorum) on the hydration of Portland cement and the development of a new compatibility index. Cement and Concrete Composites, v.30, p.37-43, 2008.

PALUDZYSZYN FILHO, E.; SANTOS, P. E. T.; FERREIRA, C. A. Eucaliptos indicados para plantio no estado do Paraná. Colombo: Embrapa Florestais, 2006. 45p. (Documentos, 129)

PARCHEN, C. F. A. Compósito madeira cimento de baixa densidade produzido com compactação vibro dinâmica. 2012. 172f. Tese (Doutorado em Ciências Florestais) Universidade Federal do Parará, Curitiba, 2012.

PEREIRA, J. C. D. et al. Características da madeira de algumas espécies de eucalipto plantadas no Brasil. Colombo: Embrapa Florestais, 2000. 113p. (Documentos, 38)

PIRRAGLIA, A. et al. Fuel properties and suitability of Eucalyptus benthamii and Eucalyptus macarthurii for torrefied wood and pallets. Bioresources, v.7, n.1, p.217-235, 2012.

SEMPLE, K. E.; CUNNINGHAM, R. B.; EVANS, P. D. Compatibility of eight temperate Australian Eucalyptus species with Portland Cement In: EVANS, P. D. Wood-cement composites in the Asia-Pacific region. Cambera: ACIAR Proceedings, 2002a. p.40-46.

SEMPLE, K. E.; CUNNINGHAM, R. B.; EVANS, P. D. The suitability of five Western Australian mallee eucalypt species for wood-cement composites. Industrial Crops and Products, v.16, n.1, p.89-100, 2002b.

WEATHERWAX, R. C.; TARKOW, H. Effect of wood on setting of Portland Cement. Forest Product Journal, v.14, n.12, p.567-570, 1964.

ZUCCO, L. L. Estudo da viabilidade de fabricação de placas de compósitos à base de cimento e casca de arroz. 1999. 133f. Dissertação (Mestrado em Engenharia Agrícola) - Unicamp, Campinas, 1999. 